\title{
Bientôt une étude pilote pour le premier dépistage génétique ò la naissance en France !
}

$\mathrm{U}$ $\mathrm{n}$ programme national de dépistage néonatal permet déjà en France de rechercher plusieurs maladies, dont la phénylcétonurie, l'hypothyroïdie, la mucoviscidose ou la drépanocytose, pour ne citer que les plus emblématiques d'entre elles. Le dépistage est réalisé au moyen de tests biologiques ou par spectrométrie de masse à partir d'une goutte de sang recueillie sur papier buvard dans les premiers jours de vie. Le troisième Plan national maladies rares, qui couvre la période 2018-2022, prévoit d'augmenter le nombre des pathologies dépistées à la naissance. Le choix obéit à des règles et des critères précis et nécessite une validation par la Haute Autorité de Santé.

Le principe fondateur du dépistage néonatal est d'identifier, parmi tous les nouveau-nés, ceux susceptibles d'être atteints d'une maladie grave et qui, bénéficiant d'un diagnostic dans les premiers jours de vie, auront accès à un traitement précoce, ou une prise en charge capable de modifier le cours de leur maladie, avant même que n'apparaissent des lésions irréversibles. Tout dépistage positif doit conduire à la prise en charge immédiate du nouveau-né pour confirmation diagnostique et, selon les cas, instauration de mesures de prévention secondaires pour empêcher la survenue des complications ou d'un traitement qui atténue les conséquences de la maladie et améliore le pronostic.

L'amyotrophie spinale proximale liée au gène SMN1 (ou SMA pour spinal muscular atrophy) bénéficie désormais de plusieurs traitements efficaces. Dans ce contexte, son dépistage à la naissance prend tout son sens. Cette maladie à transmission autosomique récessive, caractérisée par une dégénérescence des motoneurones de la moelle épinière, est la cause génétique la plus fréquente de mortalité chez l'enfant. La SMA est liée dans 90 à $98 \%$ des cas à une délétion homozygote du gène SMN1. Il existe un autre gène, SMN2, proche du gène SMN1, dont le nombre de copies est inversement corrélé à la sévérité clinique de la maladie. Des médicaments innovants, ciblant l'épissage des ARN messagers de SMN2 ou visant à remplacer SMN1, ont été récemment développés. Les résultats des nombreux essais et études cliniques convergent pour montrer qu'un traitement précoce, dans l'idéal à un stade pré-symptomatique, est beaucoup plus efficace qu'un traitement administré au stade symptomatique, lequel améliore néanmoins les facultés motrices de l'enfant. Au vu des données cliniques disponibles, les experts s'accordent actuellement au niveau mondial sur l'utilité d'un dépistage néonatal de la SMA pour optimiser le bénéfice clinique de ses traitements. Mais traiter des patients le plus souvent pré-symptomatiques implique de pouvoir les identifier de manière fiable à grande échelle et de mettre en ouvre efficacement, et dans les meilleurs délais, un traitement. Des études pilotes, toutes basées sur une recherche des délétions homozygotes de SMN1 en période néonatale, ont déjà été menées à travers le monde, notamment à Taiwan, en Belgique, en Allemagne, en Italie et en Australie, avec comme objectif commun un déploiement à terme à l'échelle nationale. Elles ont confirmé la faisabilité technique du dépistage néonatal de la SMA et de son bien-fondé. Plusieurs études pilotes concluantes ont également eu lieu aux États-Unis. Ainsi, depuis mai 2017, des recommandations fédérales proposent aux États américains qui le souhaitent de rajouter la SMA à la liste des maladies recommandées au dépistage. À ce jour, 23 États ont adopté et réalisent ce dépistage néonatal, quatorze l'ont adopté et sont en cours de déploiement et trois en sont encore au stade de pilote. Très récemment et plus proche de nous, l'Allemagne vient de décider l'implémentation du dépistage de la SMA au niveau national.

En France, l'AFM-Téléthon, la filière nationale FILNEMUS, les Hôpitaux Universitaires de Strasbourg et le $\mathrm{CHU}$ de Bordeaux travaillent ensemble à une étude pilote dédiée (DEPISMA), sur deux régions : le Grand Est et la Nouvelle Aquitaine. Elle s'appuiera sur des structures régionales existantes : centres de référence labellisés pour les maladies neuromusculaires, centres régionaux de dépistage néonatal, réseaux régionaux de périnatalité et laboratoires de génétique moléculaire. La faisabilité à grande échelle du dépistage SMA restera à démontrer. Il s'agira du tout premier dépistage néonatal utilisant une technique de génétique moléculaire dans notre pays, les autres dépistages faisant appel à des techniques biochimques traditionnelles. Ce type de dépistage néonatal génétique n'était jusqu'ici pas possible en France du fait des conditions d'encadrement très strictes en matière 
d'investigation génétique humaine. La dernière révision de la loi de bioéthique vient de l'autoriser et il est prévu que le retour d'expérience de l'étude pilote DEPISMA pourra aider à la rédaction du décret d'application de l'article concerné. La finalisation du protocole et son dépôt devant un comité de protection des personnes sont prévus au deuxième semestre 2021, pour un démarrage opérationnel envisagé dès le premier trimestre 2022.

Soon a pilot study for the first genetic neonatal screening in France!

Caroline Stalens, Christian Cottet AFM-Téléthon, Évry, France Caroline Espil-Taris CHU Bordeaux, France

Vincent Laugel Hôpitaux Universitaires de Strasbourg, France
LIENS D'INTÉRÊT

Les auteurs déclarent n'avoir aucun lien d'intérêt concernant les données publiées dans cet article.

\begin{tabular}{|c|c|c|}
\hline \multicolumn{2}{|c|}{$\begin{array}{l}\text { MYOBASE.ORG } \\
\text { LE PORTAIL DOCUMENTAIRE SUR LES MALADIES NEUROMUSCULAIRES }\end{array}$} & AFMTELETHON \\
\hline \multicolumn{3}{|c|}{$\begin{array}{l}\text { Ce portail documentaire spécialisé dans le domaine des maladies } \\
\text { neuromusculaires, les situations de handicap qu'elles génèrent et leurs aspects } \\
\text { psychologiques est publié par l'AFM-Téléthon. }\end{array}$} \\
\hline UN ACCÉS facile et simple & $\begin{array}{l}\text { Un outil ergonomique, UNE } \\
\text { INTERFACE BILINGUE }\end{array}$ & $\begin{array}{l}\text { En 2019, Myobase } \\
\text { donne un accès }\end{array}$ \\
\hline $\begin{array}{l}\text { - Utiliser les guillements } \\
\text { pour la recherche d'une } \\
\text { expression } \\
\text { "maladie de pompe" } \\
\text { - Utiliser le signe + pour } \\
\text { signifier ET } \\
\text { "sma?+mutation" }\end{array}$ & $\begin{array}{l}\text { - Laissez-vous guider par les } \\
\text { tutoriels } \\
\text { - Lancez une recherche et } \\
\text { affinez votre sélection grâce } \\
\text { aux filtres }\end{array}$ & $\begin{array}{l}\text { libre à } 75 \% \text { des } \\
55000 \text { notices } \\
\text { bibliographiques } \\
\text { du fond documentaire } \\
\text { collecté depuis } 1990\end{array}$ \\
\hline $\begin{array}{l}\text { - Utiliser le signe - pour } \\
\text { signifier NON } \\
\text { "Duchenne--diagnostic" }\end{array}$ & $\begin{array}{l}\text { TOUT MYOBASE } \\
\text { Recherchet. } \quad \text { Ok } \\
\text { Rechorche avancie }\end{array}$ & $\begin{array}{l}\text { > articles de la littérature } \\
\text { biomédicale } \\
\text { et psycho-sociale }\end{array}$ \\
\hline ( $:$ barre espace) & 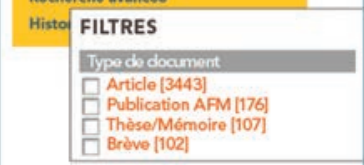 & $\begin{array}{l}\text { > livres, thèses... } \\
\text { > guides d'associations } \\
\text { et rapports } \\
\text { institutionnels } \\
\text { d'agences }\end{array}$ \\
\hline $\begin{array}{l}\text { - } \\
\text { - Cliquez sur l'onglet } \\
\text { thématique qui vous } \\
\text { convient (haut de la page } \\
\text { d'accueil) }\end{array}$ & 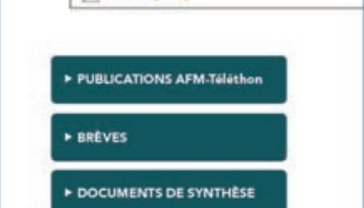 & $\begin{array}{l}\text { internationales } \\
\text { > brèves en français, } \\
\text { synthèses des articles } \\
\text { médico-scientifiques } \\
\text { internationaux les plus } \\
\text { pertinents }\end{array}$ \\
\hline $\begin{array}{l}\text { - Abonnez-vous aux alertes } \\
\text { thématiques. Créez vos } \\
\text { alertes personnalisées en } \\
\text { ouvrant un compte } \\
\text { personnel } \\
\text { - Téléchargez la Veille } \\
\text { Neuromusculaire } \\
\text { - Abonnez-vous aux flux RSS }\end{array}$ & $\begin{array}{l}\text { - instint des Biotherapies } \\
\text { Pubucations } \\
\text { Partagez les résultats } \\
\text { de votre recherche } \\
\text { - Utilisez les onglets } \\
\text { thématiques ou type } \\
\text { de publication }\end{array}$ & $\begin{array}{l}\text { > publications } \\
\text { AFM-Téléthon } \\
\text { destinées aux } \\
\text { professionnels de } \\
\text { santé ou aux personnes } \\
\text { atteintes de maladie } \\
\text { neuromusculaire et } \\
\text { à leur entourage }\end{array}$ \\
\hline
\end{tabular}

